\title{
A Three-Year Analysis of the Impact of a Student-Run Gynecology Clinic on Access to Reproductive Health Care for Uninsured Women in East Harlem
}

\author{
Eileen Wang ${ }^{1} \cdot$ Chloe Getrajdman $^{1}$ (D) Gabriela Frid ${ }^{1} \cdot$ Japjot Bal $^{1} \cdot$ Cynthia Abraham ${ }^{2} \cdot$ Adam Jacobs $^{2}$. \\ Yasmin Meah ${ }^{2} \cdot$ Farida Nentin ${ }^{1,2}$
}

Accepted: 7 May 2021 / Published online: 13 May 2021

(c) The Author(s), under exclusive licence to Springer Science+Business Media, LLC, part of Springer Nature 2021

\begin{abstract}
The East Harlem Health Outreach Partnership (EHHOP) is a medical student-run and attending-supervised clinic that provides primary care to predominantly Spanish-speaking, uninsured patients living in East Harlem, New York. In 2010, the clinic launched a Women's Health Clinic (WHC), to offer comprehensive gynecologic and reproductive healthcare under the guidance of faculty gynecologists. In this cross-sectional study, we analyzed WHC data from January 2018 to March 2021. Over this period, 59 individual patients were seen over 39 clinical sessions through a total of 164 clinical encounters staffed by 43 medical students and 19 faculty preceptors from the Department of Obstetrics and Gynecology at Mount Sinai. The most common reasons for referral to the EHHOP WHC were abnormal uterine bleeding, contraception counseling, and management of abnormal Pap smears; the most common procedures performed were Pap smears, long-acting reversible contraception placements and removals, and colposcopies. We discuss the critical role that student-run, physician-supervised reproductive health clinics play in reducing disparities in gynecologic care for uninsured women.
\end{abstract}

Keywords Free clinic $\cdot$ Gynecology clinic $\cdot$ Reproductive health $\cdot$ Student-run clinic $\cdot$ Uninsured

\section{Introduction}

Significant disparities exist in access to gynecologic services for uninsured women across the US. Uninsured women are less likely to utilize preventative health services in general and more likely to utilize emergency care compared to women with insurance [1,2]. Uninsured women are also less likely to utilize preventative reproductive health care services including routine breast and cervical cancer screening $[3,4]$. While policy changes including the Affordable Care Act and extension of postpartum and post-abortal Medicaid coverage have expanded access to reproductive care for many women across the country, non-pregnant women who are ineligible for insurance must resort to a limited safety-net

Chloe Getrajdman

cgetrajdman@gmail.com

1 Icahn School of Medicine at Mount Sinai, 1 Gustave L. Levy Pl, NY 10029 New York, USA

2 The Mount Sinai Hospital, 1468 Madison Ave, NY 10029 New York, USA for care that often lacks resources and infrastructure for longitudinal access [5].

Free clinics, including student-run free clinics, provide care to a substantial number of underserved populations throughout the United States [6]. According to one study, an estimated 1.8 million individuals in the United States receive care from free clinics annually [7]. While many of these clinics are focused on primary care and managing common chronic diseases, a smaller proportion have robust gynecologic and reproductive health access. While approximately half of free clinic patients are women, limited health-related data exist for female free clinic patients. One study estimated that female free clinic patients reported lower health-related quality of life on all aspects of women's health compared to the US baseline scores, and were less likely to utilize preventative care including: mammograms, pap smear and HPV vaccination compared to the US general population [8]. Moreover, there remains limited literature on the contributions of student-run gynecologic clinics to reproductive health care access for uninsured and underinsured women in the in the United States. 
The East Harlem Health Outreach Partnership (EHHOP) Women's Health Clinic (WHC) is a student-run and attending-supervised free clinic for adult women who do not qualify for insurance and reside in East Harlem. The EHHOPWHC cohabits with the primary care and mental health clinics; the mission is to provide comprehensive women's health care for women in the East Harlem community who also receive primary, preventative and mental healthcare within EHHOP. Staff physicians and a dedicated student team also provide consultative support for student and faculty practitioners in the primary care clinic. In this paper, we report on the demographics and clinical concerns of women referred to the EHHOP-WHC, as well as the breadth and frequency of services provided at the clinic and clinical outcomes of the patient population.

\section{Methods}

\section{Operational Structure of EHHOP-WHC}

EHHOP is a free, student-run and attending-supervised clinic that provides primary health care to more than 300 adult patients per year who live in East Harlem and the South Bronx. The EHHOP WHC functions as an ancillary clinic, to which patients are referred by the EHHOP primary care clinic, and it is run monthly on Saturdays at a gynecology ambulatory clinic associated with the Mount Sinai Hospital Department of Obstetrics and Gynecology. One chief gynecology attending and one "Chief Teaching Senior," a fourth-year medical student, oversee the overall operations of the clinic. Two to three additional fourth-year medical students, referred to as "Teaching Seniors," share the role of organizing the clinical sessions, recruiting student and attending volunteers via email listserv, following-up on lab results, communicating with patients, and developing plans of care in collaboration with the WHC attendings throughout the year. One Teaching Senior takes charge of each clinic month. During the specified clinic day, two to three "Senior Clinician" volunteers, typically third- and fourth-year students who have completed their third-year clerkship in Obstetrics and Gynecology, provide gynecological care. They are supervised directly by two to three attending volunteers affiliated with the Mount Sinai Health System. Fellows or fourth-year residents may also act as preceptors, although with the supervision of the chief attending. Finally, "Junior Clinician" volunteers, generally first- and second-year students, also are recruited to accompany and learn from the Senior Clinicians in their visits.

In addition to clinicians, one of two "Clinic Managers," generally first- or second- year medical or master's students, manage scheduling for the week and are tasked with putting patients into the electronic medical record system and calling with appointment reminders. They are also present on clinic day to check in patients upon arrival and schedule follow up appointments. All established patients are given a clinic phone number which they can call at any time, and through a voicemail system managed by the Clinic Managers, messages are forwarded to the Chief Teaching Senior within $24 \mathrm{~h}$ and are triaged accordingly.

On average, 6 patients are scheduled per clinic, 4 full visits which are allotted an hour and a half, and two quick visits which are allotted $30 \mathrm{~min}$. Full visits generally address multiple problems and can involve prolonged and/ or multiple procedures such as colposcopy or endometrial biopsy, while quick visits address one issue and can include one short procedure such as a Pap smear. On clinic day, the Teaching Senior first gives a 30-minute didactic on a gynecology topic to the Senior and Junior Clinicians, assigns patients to each Senior Clinician and then goes over the sign-ins. Sign-ins are created by the Teaching Senior in charge of that month's clinic, reviewed by the Chief Teaching Senior, and include detailed problem lists, anticipated procedures or labs, and are meant to guide the Senior Clinician in how to structure the visit. When the patient arrives, the Senior Clinicians take a history and obtain vitals on patients, before precepting with the attending and going back together to perform the physical exam or procedure, including education and consent. The Senior Clinician will then provide the plan and any further education to the patient. The Senior Clinician conducts all procedures in conjunction with $\mathrm{OB} / \mathrm{GYN}$ faculty; these include long-acting reversible contraception (LARC) placement, colposcopy, endometrial biopsy and Pap smears. The supervising physician is physically present during the entire procedure providing direct feedback in real-time and ensuring safe, high quality and effective practice and patient comfort.

Blood and urine samples are collected by the Teaching Senior who will bring the samples, including pathology and cytology samples, to their respective laboratories at the end of the clinic day. Throughout the subsequent weeks as the tests results become available, the Teaching Senior will review the results, come up with an assessment and plan including any follow-up testing that needs to be performed, and review the plan with the Chief Attending who will counsel, modify and sign-off on the finalized plan. The Teaching Senior is also responsible for calling all patients with their results; the attending physician is available to supervise and offer additional counselling, when necessary. During the weeks following clinic, the Chief Attending also signs orders for prescriptions and referrals that are needed, including those to radiology and hospital subspecialty clinics, such as colposcopy/LEEP clinic or the gynecologic surgery booking clinic. A dedicated "Access to Care Team" made up of first- and second-year medical students schedule all referrals 
and coordinate with the patients and Teaching Seniors to ensure that referral visits are completed.

Given that EHHOP WHC takes place in the hospital gynecology clinic, all supplies and equipment are provided on-site, and the cost of the supplies (with the exception of LARC devices) is covered by the hospital. The EHHOP clinic is supported by grants and donations which allow for free medication coverage. LARC devices are specifically covered by a grant established by the Family Planning Division that funds Mirena, Liletta, and Paragard IUDs as well as Nexplanon implants for patients at Mount Sinai who are unable to pay for such devices. Surgical procedures and imaging are also provided at no cost to patients through a robust social work program that allows for patients to apply for Emergency Medicaid in collaboration with a charity care program at Mount Sinai Hospital. For example, breast imaging including mammography with tomography, MRIs, ultrasounds and biopsies are provided by the Dubin Breast Health Center of Mount Sinai at no out-of-pocket cost to patients. Patients receive such services through the hospital's charity care program and grant funds that support breast outreach. When EHHOP patients become pregnant, they are immediately referred to the hospital obstetric practice where they are connected with a social worker at the first visit who will help the patient apply for Emergency Medicaid. In New York City, pregnant women who were previously uninsured and do not otherwise qualify for insurance, are covered while they are pregnant until 6 weeks postpartum. After the 6-week postpartum visit, women are then seen back at the EHHOP-WHC.

\section{EHHOP WHC Patient Demographics and Clinic Composition}

A retrospective chart review identifying the demographics, number of encounters, types of medical and gynecologic problems, and services provided by the EHHOP WHC from January 2018 to March 2021 was conducted. Due to the COVID-19 pandemic, there were no EHHOP-WHC clinic sessions from April-June 2020. The demographic data and clinical outcomes are reported using descriptive statistics. Medians and interquartile ranges (IQRs) are used to summarize data.

Over 36 months, we conducted 39 clinic sessions for 59 unique patients through a total of 164 clinical encounters. During this time period, 49 unique medical students served as Senior Clinicians and 23 unique attendings served as preceptors. WHC patients on average attended 2 visits over this time period. The overall no-show rate was $19 \%$.

Patient demographics are shown in Table 1. Patients were primarily middle-aged (median age 41 ) and multiparous (median gravidity 4). $96.6 \%$ of patients were documented as Hispanic, the majority of whom were of Mexican
Table 1 Patient and clinic demographics

\begin{tabular}{|c|c|}
\hline \multicolumn{2}{|l|}{ Patient characteristic $(n=59)$} \\
\hline $\mathrm{Age}^{\mathrm{a}}$ & $41(37-48.5)$ \\
\hline $\mathrm{BMI}^{\mathrm{a}}$ & $30.4(26.4-34.1)$ \\
\hline Obesity $^{\mathrm{b}}(\%)$ & $31(52.5)$ \\
\hline Number of completed visits per patient ${ }^{\mathrm{a}}$ & $2(1-4)$ \\
\hline Number of scheduled visits per patient ${ }^{\mathrm{a}}$ & $3(1.5-4)$ \\
\hline \multicolumn{2}{|l|}{ Ethnicity $(\%)$} \\
\hline Hispanic & $57(96.6)$ \\
\hline Non-Hispanic & $2(3.4)$ \\
\hline \multicolumn{2}{|l|}{ Nationality (\%) } \\
\hline Mexican & $45(76.3)$ \\
\hline Ecuadorian & $6(10.2)$ \\
\hline Dominican & $3(5.1)$ \\
\hline Guatemalan & $1(1.7)$ \\
\hline French & $1(1.7)$ \\
\hline Guyanese & $1(1.7)$ \\
\hline Brazilian & $1(1.7)$ \\
\hline Puerto rican & $1(1.7)$ \\
\hline \multicolumn{2}{|l|}{ Primary language (\%) } \\
\hline Spanish & $54(91.5)$ \\
\hline English & $5(8.5)$ \\
\hline Gravidity $^{\mathrm{a}}$ & $4(3-5)$ \\
\hline Prior full term deliveries ${ }^{\mathrm{a}}$ & $3(2-3)$ \\
\hline Prior preterm deliveries ${ }^{\mathrm{a}}$ & $0(0-0)$ \\
\hline Prior miscarriages or abortions $\mathrm{s}^{\mathrm{a}}$ & $1(0-1)$ \\
\hline Living children ${ }^{\mathrm{a}}$ & $3(2-3.5)$ \\
\hline \multicolumn{2}{|l|}{ Medical History (\%) } \\
\hline Anxiety/Depression/PTSD & $27(45.8)$ \\
\hline Pre-Diabetes & $15(25.4)$ \\
\hline Diabetes & $12(20.3)$ \\
\hline Hypertension & $7(11.9)$ \\
\hline Hyperlipidemia & $8(13.6)$ \\
\hline Asthma & 7 (11.9) \\
\hline Autoimmune disease & $6(10.2)$ \\
\hline Non-alcoholic fatty liver disease & $5(8.5)$ \\
\hline Chronic kidney disease & $2(3.4)$ \\
\hline Substance use disorder & $1(1.2)$ \\
\hline History of cancer & $1(1.2)$ \\
\hline Kallman’s syndrome & $1(1.2)$ \\
\hline Coronary artery disease & $1(1.2)$ \\
\hline History of intimate partner violence (\%) & $19(32.2)$ \\
\hline $\begin{array}{l}\text { Previously or currently followed by EHHOP } \\
\text { mental health clinic }\end{array}$ & $23(39.0)$ \\
\hline
\end{tabular}

${ }^{\mathrm{a}}$ Data reported as median, IQR

${ }^{\mathrm{b}}$ Defined as BMI $\geq 30.0$

heritage (76\%). Spanish was the primary language of $91.5 \%$ of patients. A substantial proportion of patients seen at WHC had pre-existing chronic conditions: $52.5 \%$ of patients had BMIs that were in the obese range; 25.4 and $20.2 \%$ had 
pre-diabetes and diabetes, respectively. A smaller proportion of patients had hypertension (11.9\%), hyperlipidemia (13.6\%), and asthma (11.9\%). Nearly $46 \%$ of WHC patients had a history of mental illness including anxiety, depression or PTSD; $32.2 \%$ of WHC patients had a history of intimate partner violence. $39 \%$ were followed concurrently by the ancillary EHHOP Mental Health Clinic.

\section{Gynecologic Needs and Services}

The numbers of gynecologic needs addressed by clinical encounter are shown in Table 2. The majority of clinical encounters addressed abnormal uterine bleeding (AUB) (43.9\%), followed by contraception counseling (39.0\%), abdominal or pelvic pain (35.4\%), and abnormal Pap smear management $(31.7 \%)$.

The numbers of procedures and laboratories performed are shown in Table 3. WHC Clinicians performed 60 Pap smears, 15 endometrial biopsies and 16 colposcopies; in addition, 15 IUDs and 4 Nexplanons were placed and 11 IUDs and 6 Nexplanons were removed; Eight Depo Provera injections were provided, and 8 oral contraceptives were prescribed for patients who opted against or were ineligible for LARCs. Our clinic also offered point-of-care ultrasound, vulvar biopsy, sexually transmitted infection and vaginitis

Table 2 Gynecologic needs addressed per clinical encounter

\begin{tabular}{ll}
\hline Gynecologic Needs (n=164 clinical encounters) & \\
\hline Abnormal uterine bleeding (AUB) (\%) & $72(43.9)$ \\
Heavy Menstrual Bleeding & $39(54.2)$ \\
Ovulatory Dysfunction & $19(26.4)$ \\
Post-Coital Bleeding & $8(11.1)$ \\
Post-Menopausal Bleeding & $6(8.3)$ \\
Contraception Counseling (\%) & $64(39.0)$ \\
Abdominal Pain/Pelvic Pain/Dyspareunia (\%) & $58(35.4)$ \\
Abnormal Pap smear management (\%) & $52(31.7)$ \\
Vaginal Discharge/Pruritis (\%) & $23(14.0)$ \\
Breast Symptoms (\%) & $21(12.8)$ \\
Pain & $14(66.7)$ \\
Discharge & $3(14.3)$ \\
Lump/Mass & $4(19.0)$ \\
Menopause Symptoms/Counseling (\%) & $11(6.7)$ \\
Vaginal/Vulvar Lesions (\%) & $11(6.7)$ \\
Intimate Partner Violence (\%) & $11(6.7)$ \\
History of Ovarian Cysts (\%) & $9(5.5)$ \\
Preconception Counseling/Infertility (\%) & $9(5.5)$ \\
Urinary Symptoms (\%) & $40(24.4)$ \\
History of Fibroids (\%) & $3(1.8)$ \\
LEEP Counseling (\%) & $3(1.8)$ \\
Gynecologic Imaging Results Reviewed (\%) & $3(1.8)$ \\
Recent Miscarriage/Abortion Counseling (\%) & $2(1.2)$ \\
\hline
\end{tabular}

Table 3 Gynecologic services provided by clinical encounter

\begin{tabular}{lr}
\hline Gynecologic Services $(\mathrm{n}=164$ clinical encounters) & \\
\hline Pap smears (\%) & $60(36.6)$ \\
IUD Placement (\%) & $15(9.1)$ \\
IUD Removal (\%) & $11(6.7)$ \\
IUD String Check (\%) & $12(7.3)$ \\
Nexplanon Placement (\%) & $4(2.4)$ \\
Nexplanon Removal (\%) & $6(3.7)$ \\
Endometrial Biopsy (\%) & $15(9.1)$ \\
Colposcopy (\%) & $16(9.8)$ \\
Point-of-care TVUS (\%) & $8(4.9)$ \\
Vulvar biopsy (\%) & $1(0.6)$ \\
Trichloroacetic acid treatment (\%) & $4(2.4)$ \\
Sexually Transmitted Infection (STI) Testing (\%) & $83(50.6)$ \\
Gonorrhea/Chlamydia & 71 \\
HIV & 44 \\
Hepatitis C & 40 \\
Hepatitis B & 31 \\
Syphilis & 35 \\
Vaginitis Testing (\%) & $13(7.9)$ \\
Candida & 6 \\
Bacterial Vaginosis & 7 \\
Trichomonas & $11(6.7)$ \\
Group B Strep & $5(3.0)$ \\
Urinalysis (\%) & 11 \\
Hormonal Testing (including fertility assessment) $(\%)$ & 1 \\
Depo Provera Administered (\%) & $26(15.9)$ \\
Hepatitis B Vaccine Administered (\%) & 11 (6.9) \\
Influenza Vaccine Administered (\%) & \\
\hline & \\
\hline
\end{tabular}

testing as well as hormonal evaluations for oligomenorrhea, menopause, polycystic ovary syndrome and infertility. To supplement the needs of an overburdened primary care clinic, vaccinations for Hepatitis B and Influenza were also provided on site.

\section{Gynecologic Outcomes and Referrals}

\section{Abnormal Papanicolaou Smear Management}

Of the 60 Pap smears performed, 22 were abnormal, which is defined as any cytology other than NILM or HPV negative, excluding insufficient samples. Of those, 14 had a positive HPV result and 16 had a positive cytology result (ASCUS, ASC-H, AGC, LSIL or HSIL). 100\% of patients with abnormal Pap smears returned to the clinic for further management. Of the 16 colposcopies performed, $11 \mathrm{dem}$ onstrated CIN 1; none showed CIN 2 or CIN 3 (Table 4).

Six referrals were made to the hospital colposcopy/LEEP clinic for further evaluation due to persistently abnormal Pap smears or concern for a high-grade lesion. Of those, four 
Table 4 Visit outcomes by patient encounter

\begin{tabular}{|c|c|}
\hline \multicolumn{2}{|l|}{ Gynecologic outcomes } \\
\hline \multicolumn{2}{|l|}{ Pap smear HPV results ${ }^{\mathrm{a}}(\%)$} \\
\hline Positive & $14(25.5)$ \\
\hline Negative & $41(74.5)$ \\
\hline \multicolumn{2}{|l|}{ Pap smear cytology results (\%) } \\
\hline NILM & $33(55.0)$ \\
\hline ASCUS & $9(15.0)$ \\
\hline ASC-H & $3(5.0)$ \\
\hline LSIL & $2(3.3)$ \\
\hline HSIL & $1(1.7)$ \\
\hline AGC & $1(1.7)$ \\
\hline Insufficient sample/Sample unable to be process/Sample lost & $11(18.3)$ \\
\hline \multicolumn{2}{|l|}{ Endometrial biopsy result (\%) } \\
\hline Benign/unremarkable & $11(73.3)$ \\
\hline Endometrial polyp & $3(20.0)$ \\
\hline Chronic endometritis & $1(6.7)$ \\
\hline \multicolumn{2}{|l|}{ Colposcopy results (\%) } \\
\hline No biopsies performed & $2(12.5)$ \\
\hline Negative for CIN & $3(18.8)$ \\
\hline CIN1 & $11(68.8)$ \\
\hline \multicolumn{2}{|l|}{ Vaginitis testing positive results (\% positive) } \\
\hline Candida & $0(0)$ \\
\hline Bacterial vaginosis & $2(20)$ \\
\hline Trichomonas & $0(0)$ \\
\hline Group B Strep & $0(0)$ \\
\hline \multicolumn{2}{|l|}{ Vulvar biopsy result } \\
\hline HPV condyloma (HPV 43) & $1(100)$ \\
\hline \multicolumn{2}{|l|}{ STI testing positive results (\% positive) } \\
\hline Chlamydia & $2(2.8)$ \\
\hline Gonorrhea & $0(0)$ \\
\hline HIV & $0(0)$ \\
\hline Hepatitis C & $0(0)$ \\
\hline Hepatitis B & $1(3.2)$ \\
\hline Syphilis & $0(0)$ \\
\hline Urinalysis positive for urinary tract infection (\% positive) & $4(15.4 \%)$ \\
\hline PCOS diagnosis made $(\%)$ & $2(66 \%)$ \\
\hline Encounters during which medications were prescribed (\%) & $51(31.1)$ \\
\hline \multicolumn{2}{|l|}{ Types of medications prescribed } \\
\hline Antibiotics & 20 \\
\hline Anti-fungal (Candidiasis) & 9 \\
\hline Azithromycin (Chlamydia) & 2 \\
\hline Metronidazole (Bacterial vaginosis) & 2 \\
\hline Doxycycline (Chronic endometritis) & 1 \\
\hline Nitrofurantoin/TMP-SMX (Urinary tract infection) & 6 \\
\hline Oral progesterone & 9 \\
\hline Norethisterone & 3 \\
\hline Medroxyprogesterone & 6 \\
\hline Combined oral contraception & 5 \\
\hline Vaginal estrogen cream/Lubrication & 9 \\
\hline Lactobacillus probiotic & 2 \\
\hline Prenatal vitamins & 2 \\
\hline Clomiphene citrate & 1 \\
\hline
\end{tabular}

a Among those Pap smears in which co-testing was performed and result obtained elected conservative management after counseling and two underwent LEEP; those two excisional biopsy pathologies showed one CIN 1 with negative margins and one CIN 3 with positive margins.

\section{Management of Abnormal Ultrasounds}

Transvaginal ultrasound (TVUS) was available on site; for those patients requiring the expertise of a radiologist for definitive imaging, EHHOP-WHC referred such patients to Mount Sinai Radiology. Twenty-nine patients underwent a TVUS through Mount Sinai Radiology; 14 of these cases demonstrated an abnormality that required gynecologic follow-up, biopsy or a repeat interval ultrasound. Of these, 5 patients were referred to gynecology surgery clinic-4 for AUB and 1 for a complicated IUD removal. One patient underwent hysterectomy and ovarian cystectomy for heavy uterine bleeding that required intravenous iron. Surgical pathology for this patient revealed adenomyosis and multiple leiomyomas, as well as one benign serous cystadenoma and one hemorrhagic corpus luteal cyst.

\section{Mammography}

47 mammogram referrals were made for $30 \mathrm{WHC}$ patients over this 3 -year time period. 3 patients had a BIRADS of 3 or greater, 6 required further breast imaging after mammogram and 3 underwent breast biopsy that demonstrated fibroadenoma, nodular fibrous benign breast tissue with pseudoangiomatous stromal hyperplasia, and invasive poorly differentiated duct carcinoma. The patient diagnosed with breast cancer went on to follow-up with an oncologist and received chemotherapy. 10 patients also underwent breast ultrasound for dense breasts, further evaluation of mammogram results or breast lump, discharge or pain. Of note, 12 out of 12 patients greater than age 50 seen at WHC had a screening mammogram within the last 2 years indicating $100 \%$ compliance with the United States Preventive Services Task Force (USPSTF) guidelines.

\section{Discussion}

Prior literature on safety-net and uninsured populations have shown that student-run free clinics (SRFCs) can play an important role in increasing access to preventative services such as cervical cancer and breast cancer screening [9, 10]. However, dedicated gynecology clinics such as EHHOP WHC offer the time and space to address other women's health complaints with robust systems for referral to subspecialists, such as abnormal Pap smear management, AUB and contraception-among the top gynecologic needs in our uninsured population. EHHOP WHC is unique as a 
student-run clinic insofar as it also offers same-day procedures such as colposcopy, endometrial biopsy and point-ofcare transvaginal ultrasound, allowing for a quicker, more streamlined care, and broader range of diagnostic and management options for our patient population. The WHC was a major resource to the primary care clinic in identifying and managing abnormal Pap smears requiring closer monitoring, and in some cases invasive testing and surgical management. The WHC also offered substantial support for contraception, with robust access to LARCs through our partnerships with the Family Planning Division, including IUD and Nexplanon insertions and Depo Provera injections, that are not usually available at student-run free clinics. LARCs are highly-effective, long-term options for contraception, as well as treatment options for abnormal uterine bleeding and pelvic pain, but continue to be underutilized in low-income women in the US due to the high cost and limited access to the devices [11]. Making LARCs available on-site, sameday, and free-of-charge at a SRFC like EHHOP decreases time, cost, logistical challenges of obtaining them and allows uninsured women the full range of available contraceptive methods and gynecology treatments, however we acknowledge this may not be feasible in other student-run or safety net clinics. EHHOP WHC also offered advice on other contraceptive options for those for whom LARCs are contraindicated or not opted.

Pelvic pain was a predominant complaint for which patients were referred to WHC; access to point-of-care TVUS as well as expedited referral for official TVUS scans allowed for quick and thorough work-up of pelvic pain with options for hormonal treatment including IUD insertion when appropriate. Certain services, such as abortions and infertility treatment, were not offered at WHC but easily accessed through a facilitated referral network.

Our results also emphasize the importance of interdisciplinary collaboration for uninsured populations. The majority of our WHC patients tend to have chronic conditions that were being followed at the main primary care clinic, such as hypertension, diabetes and obesity, all of which have an interrelated impact on obstetrical and gynecological outcomes. In addition, a substantial number of them reported a history of IPV, which is associated with physical health consequences including sexually transmitted infections, vaginal bleeding, pelvic pain, and dyspareunia [12]. Women's health clinics may uniquely provide a safe space to not only screen for IPV but also provide trauma-informed care and address any gynecological issues associated with sexual, physical, emotional and reproductive abuse and coercion [13, 14]. At the same time, our results demonstrate the importance of student-run gynecological clinics' integration into a comprehensive primary care structure, with access to resources such as sexual assault and violence intervention programs, as well as mental health clinics.
We note that patients at WHC have lower missed encounter rates and higher follow-up rates than other student-run clinics and even non-student-run gynecology clinics [15, 16]. The fact that $100 \%$ of patients followed-up on any abnormal Pap smears and all women aged 50 or older received screening mammograms may be due to a number of factors, including a robust clinic management and an Access to Care Team that scheduled referrals and ensured minimal or no cost to patients.

Finally, we acknowledge the impact of a SRFC women's health clinic in educating students through a gynecology lens. The majority of OB/GYN education occurs in a resource-heavy inpatient setting with less emphasis on outpatient or primary care $[17,18]$. SRFC gynecology clinics offer more robust teaching to students about ambulatory women's health, with the ability to experience procedures and decision-making on common problems in the primary care setting, including contraception counseling, LARC placement, AUB management and breast health.

Future directions for the clinic include expanding subspecialty clinics such as uro-gynecology and pelvic pain, which are common concerns among our population. Comprehensive literature on quality of care and patient experience at SFRCs is limited, and particularly for gynecological care $[19,20]$. While our missed encounter and follow-up rates may give us some insight into patient satisfaction, we were not able to fully capture this in our data. Future studies should investigate this area. This study also focused on the services provided to our EHHOP patients; future research should also investigate how this parallels the student and physician volunteer experience, satisfaction with learning, as well as influence over specialty selection or preparation for residency. Finally, future efforts should address the potential for EHHOP-WHC to integrate women's health issues into the EHHOP primary care clinic, including screening for intimate partner violence and gynecological issues, as well as incorporating gynecologists as co-preceptors in the main clinic to augment women's health education among primary care providers and students.

\section{Conclusions}

The EHHOP Women's Health Clinic is an example of a student-run and attending-supervised free clinic that provides access to comprehensive women's health care for East Harlem women who are otherwise not eligible for insurance. Through a partnership with Mount Sinai hospital that allows for free medication, imaging services and surgical management, and a clinic model in which women's health care is intimately connected to primary and mental health care services, patients are able to receive interdisciplinary care with high rates of treatment adherence and follow-up. The 
results of this paper demonstrate the importance and potential of student-run subspecialty clinics in providing access to gynecologic care, as well as the role that medical students can play in expanding services to underserved populations.

Authors' Contributions EW and CG contributed to the study conception and design. Material preparation, data collection, and analysis were performed by EW, CG, GF, JB. The first draft of the manuscript was written by EW and CG and all authors commented on previous versions of the manuscript. All authors read and approved the final manuscript.

Funding Not applicable.

\section{Declarations}

Conflict of interest All authors declare that they no have conflict of interest.

\section{References}

1. Nicholson, W. K., Ellison, S. A., Grason, H., \& Powe, N. R. (2001). Patterns of ambulatory care use for gynecologic conditions: a national study. American Journal of Obstetrics and Gynecology, 184(4), 523-530. https://doi.org/10.1067/mob.2001. 111795.

2. Taylor, Y. J., Liu, T.-L., \& Howell, E. A. (2019). Insurance differences in preventive care use and adverse birth outcomes among pregnant women in a medicaid nonexpansion state: a retrospective cohort study. Journal of Women's Health, 29(1), 29-37. https:// doi.org/10.1089/jwh.2019.7658.

3. Akers, A. Y., Newmann, S. J., \& Smith, J. S. (2007). Factors underlying disparities in cervical cancer incidence, screening, and treatment in the United States. Current Problems in Cancer, 31(3), 157-181. https://doi.org/10.1016/j.currproblcancer.2007.01.001.

4. Holden, C. D., Chen, J., \& Dagher, R. K. (2015). Preventive care utilization among the uninsured by race/ethnicity and income. American Journal of Preventive Medicine, 48(1), 13-21. https:// doi.org/10.1016/j.amepre.2014.08.029.

5. Gusmano, M. K., Fairbrother, G., \& Park, H. (2002). Exploring the limits of the safety net: community health centers and care for the uninsured. Health Aff (Millwood), 21(6), 188-194. doi:https:// doi.org/10.1377/hlthaff.21.6.188.

6. Geller, S., Taylor, B. M., \& Scott, H. D. (2004). Free clinics helping to patch the safety net. Journal of Health Care for the Poor and Underserved, 15(1), 42-51. https://doi.org/10.1353/hpu.2004. 0005 .

7. Darnell, J. S. (2010). Free clinics in the United States: a nationwide survey. Archives of Internal Medicine, 170(11), 946-953. https://doi.org/10.1001/archinternmed.2010.107.

8. Kamimura, A., Myers, K., Ashby, J., Trinh, H. N., Nourian, M. M., \& Reel, J. J. (2015). Women in free clinics: an assessment of health-related quality of life for prevention and health education. Journal of Community Health, 40(4), 793-801. https://doi.org/10. 1007/s10900-015-0002-8.

9. Khalil, S., Hatch, L., Price, C. R., et al. (2020). Addressing breast cancer screening disparities among uninsured and insured patients: a student-run free clinic initiative. Journal of Community Health, 45(3), 501-505. https://doi.org/10.1007/ s10900-019-00767-x.

10. Price, C. R., Hatch, L. A., Radisic, A., et al. (2020). Enhancing adherence to cervical cancer screening guidelines at a student-run free clinic. Journal of Community Health, 45(1), 128-132. https:// doi.org/10.1007/s 10900-019-00724-8.

11. Beeson, T., Wood, S., Bruen, B., Goldberg, D. G., Mead, H., \& Rosenbaum, S. (2014). Accessibility of long-acting reversible contraceptives (LARCs) in federally qualified health centers (FQHCs). Contraception, 89(2), 91-96. https://doi.org/10.1016/j. contraception.2013.09.014.

12. Campbell, J., Jones, A. S., Dienemann, J., et al. (2002). Intimate partner violence and physical health consequences. Archives of Internal Medicine, 162(10), 1157-1163. https://doi.org/10.1001/ archinte.162.10.1157.

13. ACOG. (2012). ACOG Committee Opinion 518: Intimate Partner Violence. Obstetrics, \& Gynecology.https://www.acog.org/clini cal/clinical-guidance/committee-opinion/articles/2012/02/intim ate-partner-violence.

14. Clark, L. E., Allen, R. H., Goyal, V., Raker, C., \& Gottlieb, A. S. (2014). Reproductive coercion and co-occurring intimate partner violence in obstetrics and gynecology patients. American Journal of Obstetrics and Gynecology, 210(1), 42. . https://doi. org/10.1016/j.ajog.2013.09.019.

15. Kumar, N. R., DuVernois, G., Almeida-Monroe, V., Siegert, N., \& De Groot, A. S. (2019). Evaluating the impact of a studentrun women's clinic on access to gynecologic care for uninsured women in Rhode Island. Rhode Island Medical Journal (2013), 102(10), 52-56.

16. Miller, S. M., Tagai, E. K., Wen, K.-Y., et al. (2017). Predictors of adherence to follow-up recommendations after an abnormal Pap smear among underserved inner-city women. Patient Education and Counseling, 100(7), 1353-1359. https://doi.org/10.1016/j.pec. 2017.01.020.

17. Spencer, A. L., \& McNeil, M. (2009). Interdisciplinary curriculum to train internal medicine and obstetrics-gynecology residents in ambulatory women's health: adapting problem-based learning to residency education. J Womens Health (Larchmt) 18(9), 13691375. https://doi.org/10.1089/jwh.2008.1253

18. Norris, T. E., Schaad, D. C., DeWitt, D., Ogur, B., Hunt, D. D., \& Consortium of Longitudinal Integrated, C. (2009) Longitudinal integrated clerkships for medical students: an innovation adopted by medical schools in Australia, Canada, South Africa, and the United States. Acad Med, 84(7): 902-907. https://doi.org/10.1097/ ACM.0b013e3181a85776.

19. Lawrence, D., Bryant, T. K., Nobel, T. B., Dolansky, M. A., \& Singh, M. K. (2015). A comparative evaluation of patient satisfaction outcomes in an interprofessional student-run free clinic. Journal of Interprofessional Care, 29(5), 445-450. https://doi.org/ 10.3109/13561820.2015.1010718.

20. Meah, Y. S., Smith, E. L., \& Thomas, D. C. (2009). Student-run health clinic: Novel arena to educate medical students on systemsbased practice. The Mount Sinai Journal of Medicine, New York, 76(4), 344-356. https://doi.org/10.1002/msj.20128.

Publisher's Note Springer Nature remains neutral with regard to jurisdictional claims in published maps and institutional affiliations. 